\title{
Health technology management in Israel: HTA in action
}

\author{
Joshua Shemer, Mordechai Shani \\ Gertner Institute for Epidemiology and Health Policy Research and Tel-Aviv University

\section{Orly Tamir} \\ Gertner Institute for Epidemiology and Health Policy Research and Ariel University Center \\ in Samaria
}

\author{
Miriam Ines Siebzehner \\ Gertner Institute for Epidemiology and Health Policy Research and Tel-Aviv University
}

\begin{abstract}
Objectives: The aim of this study was to describe the history and present situation with health technology assessment (HTA) in Israel.

Methods: The method used in this study was a historical analysis based mainly on the knowledge of the authors, but supplemented by the published literature.

Results: HTA originated in Israel as a centralized function conducted under the auspice of research, developing into an active multidisciplinary center. Throughout the expansion of the field, HTA was performed in affiliation with several local and international bodies, while providing direct and indirect support at the national level. Today, mainly as a result of vigorous dissemination of the principles, methodology and tools for HTA by the Israeli Center for Technology Assessment in Health Care (ICTAHC), this discipline is increasingly a decentralized activity conducted by a great variety of institutions. Israeli health policy decisions are increasingly based on the results of HTA. Conclusions: ICTAHC's role and functioning has expanded since its beginnings. HTA has become an important part of health care in Israel.
\end{abstract}

Keywords: Health technology assessment, Israel, health technology management

The year 2008 marked the celebration of a decade since the establishment of the Israeli Center for Technology Assessment in Health Care (ICTAHC). Formally, health technology assessment (HTA) activities began 17 years ago in Israel. This study aims to summarize the recent developments in health technology management in Israel. It will also portray the evolution of Health Technology Assessment in the country, describe areas of accomplishments, and illustrate the potential research scope and projections for the future.

In 1995, the National Health Insurance Law (NHIL) was enacted in Israel (24). This act has greatly influenced the organization of the healthcare system as well as the processes underlying policy decision making at all levels of the system. The most substantial change was related to defining a National List of Health Services (NLHS) that all residents became entitled to receive from their health plans (Health Maintenance Organizations, HMOs) (6;17). All residents are insured, and they are free to choose among the four health plans, which are competing, independent, nonprofit, nongovernmental legal entities, but they operate within a legal and regulatory framework defined by the government. No permanent resident can voluntarily opt out of the NHIL system (16). There are services that are included in the NLHS and are the responsibility of the government, such as preventive health care, public health services, psychiatric care, and long-term care. Therefore, they are not provided by the health plans. Several services, however, remain outside the responsibility of both the government and the health plans like dental care, complementary medicine and optician services (3), and cosmetic plastic surgery.

The main orientations of the system are solidarity and equity while aiming at keeping high standards of care. However, the expenditures for care, and specifically, new medical technology that continually is being developed and 
adopted within the NLHS (3) are a burden to the system. The impressive and rapid development of medical technologies presents Israeli society in general, and the medical community in particular, with difficult dilemmas concerning the need and ability to implement these technologies. Certainly, these forces have stimulated the development of health technology assessment in Israel, and it evolved to be the cornerstone of health technology management of all levels in the country.

\section{DEVELOPMENT OF HTA IN ISRAEL}

The worldwide environment in the health policy arena in the early 1990s strongly encouraged the implementation of HTA with several centers emerging in different countries $(1 ; 2 ; 5 ; 7 ; 12)$. In Israel, as well, during these years, HTA themes have penetrated, a working group was created and in 1992 the Medical Technology Assessment Unit began operating. The Unit was the first official body in Israel to deal with the concept of HTA and its activation was catalyzed by two events. First, a local publication, in 1989, of guidelines for long-term national health policy (9), and second, conclusions drawn in 1990 from a thorough examination of the Israeli healthcare system that was conducted to evaluate the need for reform (28). Both works specifically mentioned the need for creating, developing, and advancing HTAas a conceptual field and as a feasible tool.

In its first years, the Medical Technology Assessment Unit focused on outlining basic principles, guidelines, and the overall framework for technology assessment. In addition, to substantiate this discipline, consideration was given to distributing the body of knowledge that was assembled and gained. Disseminating the knowledge in Israel includes, up until today, a scholastic framework taught at university as a full semester course for advanced degree students in Management of Health Systems (20), as well as sporadic invited lectures.

In 1994, the Medical Technology Assessment Unit joined ISTAHC as an active member. Later that year, the first international symposium on HTA in collaboration with ISTAHC, the Israeli Ministry of Health, and The Gertner Institute was organized in Jerusalem. Publication of a book (20) based on issues related to presentations given in the symposium followed thereafter.

Several years later, in 1998, in conjunction with a reform in the structure and tasks of the Ministry of Health, and partly as a result of a NHIL enacted in Israel in 1995, the Medical Technology Assessment Unit evolved into a national center. The center, named The Israeli Center for Technology Assessment in Health Care (ICTAHC), was launched at the Gertner Institute for Epidemiology and Health Policy Research, which is a not-for-profit institute. The center was defined as an independent research center, reflecting the expanded role granted to HTA in the country. At the same time, to allow the implementation of HTA results, the Ministry of Health founded the Medical Technology and Infrastructure
Administration as an integral organ of its medical division. The Administration was authorized to formulate policies, set standards, and regulate the adoption of medical technologies at the national level. Specifically, the Administration was appointed responsible for periodical update of the NLHS, while formally the mechanism to support this process, scientifically and academically, was developed by ICTAHC through meticulous research $(17 ; 21)$. Hence, the interaction between the Ministry of Health and ICTAHC demonstrates the use of HTA for technology management.

During the years of HTA existence in Israel, it has expanded in terms of both people involved and importance, and has widened its scope. Additional bodies began performing HTA, most of which are a result of exposure to the field and also a result of the local needs. Among these organizations are the HMOs (16), the Israeli Medical Association, health insurance companies, academic centers, health institutions, and the pharmaceutical and medical device industry. All of these bodies are engaged in HTA, which has become the main tool for technology management within their activities.

\section{HEALTH TECHNOLOGY MANAGEMENT IN ISRAEL}

HTA activities in Israel take an active place both in research and in the everyday practical setting. HTA is an essential bridge between research and practice, and there is now a strong and dual interaction between them, which allows evidence-based and systematic process for an effective technology management at all levels of the healthcare system.

\section{National Policy Level}

The most prominent manifestation of this stimulating interaction between research and practice is the process of decision making to incorporate new health technologies through public funding. For this purpose, the Medical Technology Administration at the Ministry of Health took a systematic approach of health technology assessment, based on the model suggested by Shemer and Siebzehner (21). According to this model, the Israeli mechanism for updating the NLHS is based in two main elements: HTA and decision making. HTA serves as an analytical tool. It integrates clinical, epidemiologic, and economic considerations, aiming to demonstrate the added value of each technology and the impact on the national budget. The decision-making process relies on the technologies' assessments and a set of predefined criteria, together with ethical, social, and legal considerations (29).

In Israel, every year, as part of the annual budgeting of the national healthcare system, the government allocates a predetermined budget specifically to fund new technologies. The process of updating the NLHS has been performed almost every year for more than a decade (8) with a Public National Advisory Committee (PNAC) managing its final stage. The PNAC includes twenty to twenty-five members and is appointed by the Ministers of Health and Finance. It is 
comprised by different stakeholders such as senior officials of the Ministry of Health and the Ministry of Finance, representatives of the public and the medical professions, and the four health plans, among others. This process, described and scrutinized in detail in previous studies $(6 ; 8 ; 16 ; 17 ; 22 ; 25)$, is considered by some scholars to be unique and even groundbreaking (16).

Although the updating of the NLHS is a systematic process based on HTA, there are many aspects involved, such as social, ethical, legal, and political (25). Most of the criteria are not quantifiable, which results in a true and long-lasting deliberation through the process.

One of the most prominent examples of the role of comprehensive use of HTA methods as a tool for technology management is the "Myozyme debate."

In 2006, the PNAC had reached a decision to provide public funding for the drug Myozyme (alglucosidase alfa, Genzyme Corporation), a truly "life-saving" therapy indicated for use in patients with the rare neuromuscular genetic Pompe disease. Pompe disease, is an orphan disease-in Israel, there are approximately only 10 patients out of a population of 7 million.

Committee deliberations concerning the inclusion of this therapy in the NLHS focused on the high uncertainty regarding projected prevalence of the disease and future spending on the drug (which significantly escalates in accordance to body weight increase). Furthermore, alglucosidase alfa was only recently granted marketing approval in Israel (August 2006) and in the United States (April 2006, under a priority review process), and substantial clinical evidence on the drug had not yet been collected. Recognizing the severely debilitating nature of the disease, which is usually fatal, while bearing in mind that this is an extremely costly medication beyond the reach of the individual, members of the Committee decided to support the provision of this treatment at an annual cost of 2 million NIS (\$410000) per patient (29).

Other examples involve many other criteria that are being considered as part of HTA. The addition of oncology drugs with each update of the NLHS, such as, bevacizumab (Avastin), as first-line treatment for patients with metastatic carcinoma of the colon or rectum in 2006, cetuximab (Erbitux), in combination with radiation therapy as thirdline treatment for patients with metastatic carcinoma of the colon in 2009, and many examples, demonstrate the impact of social and ethical values of life prolongation, as cancer is still considered a life-threatening disease.

Recently, the Continuous Intrathecal Baclofen Infusion Pump as a treatment option in the management of severe spasticity of cerebral origin, was included in the NLHS. This PNAC's decision reflects the criterion of improving quality of life of children suffering from cerebral palsy (10).

As a management tool, all decisions were made by committee members acting in consensus, although a recent modification in the updating mechanism enables majority voting in specific cases when consensus cannot be reached (29). At the end of its deliberations, the committee provides the final recommendations of the new technologies to be added to the NLHS, according to the allocated budget.

Although the total budget to be allocated annually is debatable, there is a consensus between policy makers and politicians that at least an additional 2 percent is required each year. This is to maintain the high level of health care and to meet professional and public demands (15).

The priority setting process is concluded after the list of prioritized technologies is approved by the government. Even though the PNAC recommendations are not binding, the committee is, in effect, the formal decision-making body on setting priorities in health technologies (18). The final list of technologies receives formal validity as an act of government and is published on the Ministry of Health Web site, and in the major daily newspapers. After governmental approval, all HMOs are required by law to provide the new technologies added to the NLHS, as an integral part of services offered to their insurees (25).

The process of updating the NLHS in Israel is the main tool to control and manage health technology. This process is unique, and not without its merits. However, the fairness and legitimacy of the priority setting mechanism have not yet been established. The main obstacles for achieving these goals may relate to the large number of technologies assessed each year within a short time frame (more than 400 technologies annually), the scarcity of personnel engaged in HTA and the desire for early adoption of new technologies (6).

\section{Health Plans Level}

The contribution of the health plans to technology management in the Israeli healthcare system is reflected within their organizational activities, as well as in their involvement in the national policy arena.

As mentioned earlier, in Israel there are four active health plans required by law to provide health services according to a NLHS, while the competition between the health plans relies, in part, on other health services, provided mostly through a complementary insurance. Almost 80 percent of the Israeli population acquired complementary insurance. For the purpose of the complementary insurance and for other purposes, all the health plans conduct HTA. Furthermore, the two largest health plans, "Maccabi" and "Clalit" Health Services, have created units that specialize in HTA, where the importance is in providing the PNAC with the health plan's position regarding the inclusion of a technology in the NLHS.

\section{Israel Medical Association}

The Israel Medical Association (IMA) incorporates all medical professions in the country and represents a point of view of the broad policy level. The IMA's approach to HTA highlights three main elements: benefit, risks, and cost of a technology. In recent years, the IMA began developing a 
mathematical computerized model, which provides a score for each technology assessed, with the assessment based on these three elements. Naturally, the IMA emphasizes especially the medical-clinical aspects of the technology. All the medical specialties are involved, and their task is to discuss and agree on the best alternatives for treatment and to choose among them.

The development of this model intends to provide an effective and rapid supportive tool for the PNAC in its priority setting of health technologies at a national level. Until the past 2 years, representatives of the IMA were part of the process of updating the NLHS led by the Ministry of Health. For the past 2 years, the IMA has started an independent activity parallel to the PNAC, although its validity and influence should be evaluated in the future.

\section{The ICTAHC}

Since its inception and throughout the years, ICTAHC has established its contribution to decisions through comprehensive work. ICTAHC remains the most influential HTA body in the country, mainly due to its strong research foundation and close interaction with all levels of organizations in the healthcare system.

The activities of ICTAHC, nowadays in particular, are performed in collaboration with the Ministry of Health, the IMA, the medical scientific professions unions, the health plans, the National Councils on Health, and various medical and academic centers.

In general, the center has four main research areas: (i) formulation of policy for health technology management, (ii) identification and early assessment of new and evolving health technologies, (iii) creation of a national platform for full economic evaluation of health technologies, and (iv) utilization patterns of health technologies.

Selected studies performed by ICTAHC over the years, all of which had a notable impact on technology-related policy issues:

Evaluation of the Process of Adoption of New Medical Technologies by the Israeli NHIL. This study evaluated the first 4 years of the national process for the inclusion of medical technologies into the NLHS. Each year, approximately 400 new health technologies were being prioritized, of which approximately fifty were fully assessed. The study findings indicated that the basic principles of the process were maintained and evolved over time. Also, the findings had spotted specific improvements that were called for, such as in the economic and epidemiological data. The process was generally accepted, with the Ministry of Health presiding as the official body that should manage and lead it. Moreover, in light of an analysis of similar activities in other countries, the process in Israel was highly appreciated by politicians, physicians, and the public, and considered by many health policy analysts in Israel and abroad to be unique and a ground braking on an international scale $(16 ; 26)$. This mechanism is currently used at a national level.

Consensus Conference on Prevention, Diagnosis, and Treatment of Osteoporosis. Due to the high prevalence of osteoporosis and high incidence of osteoporotic fractures in the elderly population in Israel, ICTAHC, in association with the Ministry of Health and the IMA, held a consensus conference that aimed to identify the scope of the problem, to analyze medical and economic aspects of the disease, and to recommend a comprehensive national policy regarding prevention, diagnosis, and treatment of osteoporosis $(23 ; 27)$. The recommendations proposed by this conference (27) were adopted "as is" by the Ministry of Health and were immediately implemented as clinical guidelines with the provision of public funding for, what was then, new therapies in osteoporosis (alendronate and raloxifene).

Consensus Conference on the Treatment and Rehabilitation of Hip Fracture. The increased incidence of hip fractures in Israel has augmented the economic burden and enlarged the workload in orthopedic and rehabilitation wards. This resulted in a need for evaluation of the required measures of improvement and resources for adequate treatment and rehabilitation. The consensus conference resulted in elucidation of updated clinical guidelines (4), which consequently led to a change in the reimbursement methodology from "per hospitalization days" to "diagnosis-related group" (11), and ultimately to a significant reduction in waiting time for surgery and in length of hospital stay (14).

Utilization Patterns of Imaging Services (Computed Tomography and Magnetic Resonance Imaging) in Israel. This study established a national database on the utilization patterns of computed tomography (CT) and magnetic resonance imaging (MRI) and the scope of such examinations in Israel. These data are updated on an ongoing basis. In light of the high level of accessibility and availability of these imaging devices in Israel, the research findings corroborated the claim that is the basis for the Certificate of Need (CON) regulations, namely, that the number of devices available for use influences the scope of utilization (13). As a result of this study, it was advised that the enacted CON regulations continue for $\mathrm{CT}$ and MRI devices in Israel, while examining the existing changes in current clinical practice.

Generating Israeli Generic Health-Related Quality of Life Tariffs. To provide a platform for an economic evaluation of cost-utility analysis, this work established health-state profiles and matched values (tariffs) representing the specific preferences of the Israeli population. The tariffs obtained through this work (Abadi-Korek et al., in process) were presented for use by any interested party, as well as by the Israeli Public National Advisory Committee, which prioritizes the technologies recommended for inclusion in the NLHS. 
Establishing a Professional and Academic Infrastructure for Short, Medium, and Long-term Forecast of New Medical Technologies. In recent years, early identification of promising technologies is emerging as a major activity of many healthcare organizations globally. With the purpose of creating a systematic mechanism for early identification of potentially effective technologies, this study reviewed international forecasting models, analyzed futuring methods, and established a model adapted to Israel to be performed on the national level (30). Several other projects are currently under way to further support technology management in the country and with the international HTA community.

Facing foreword, ICTAHC has mapped a variety of issues for research and future implementation. In general, ICTAHC continues to struggle with the formulation of a method and mechanism that will facilitate appropriate prioritization of health technologies and distribution of resources. This process embodies a wide range of perspectives that are an integral part of the HTA approach, from the selection of cost-effective interventions, to greater efficiency and more effective services. In addition, ICTAHC, from its broad point of view, is deliberating suitable means to manage the inherent tensions that arise when HTA directly influences decisions regarding health technology adoption by both the medical community and by the publicly funded health services.

Some specific issues that ICTAHC has pointed out for the future include substantiating health-states tariffs and qualityadjusted life-years as an explicit supportive tool for prioritization of medical technologies, comparing various health benefit schemes, improving forecasting methodologies, and advancing activities related to evolving technology-related trends.

\section{CONCLUSIONS}

HTA originated in Israel as a centralized function conducted under the auspice of research, developing into an active multidisciplinary center. Throughout the expansion of the field, HTA was performed in affiliation with several local and international bodies, while providing direct and indirect support at the national level. Today, mainly as a result of ICTAHC's vigorous dissemination of the principles, methodology, and tools for HTA, this discipline is increasingly a decentralized activity conducted by a great variety of organizations in the public and private sectors that make technology-related policy decisions. Yet, the decentralization of HTA activity has not been a result of a reduction in the level of centralized activity. Instead, it stems from an expansion in the activities of ICTAHC, which is reflected in the pivotal position the Center assumed and maintains within the local healthcare system throughout the years.

In summary, HTA in Israel has been fueled by industry, private, and public organizations, as well as by global trends, all pushing toward more open procedures, trans- parency, and accountability in the decision-making process regarding technology policy, and today, HTA has reached a key point not only along the diffusion curve, but also in the managerial perception of medical technology in the country.

\section{CONTACT INFORMATION}

Joshua Shemer, MD (shukis@gertner.health.gov.il), Full Professor, Department of Internal Medicine, Sackler Faculty of Medicine, Head, School of Public Health, Tel Aviv University, POB 39040, Ramat Aviv, Tel Aviv 69978, Israel; Director, Israeli Center for Technology Assessment in Health Care - ICTAHC, The Gertner Institute, Sheba Medical Center, Tel Hashomer, Ramat Gan 52621, Israel

Mordechai Shani, MD (mshani@post.tau.ac.il), Honoree, The Israel Prize for Lifetime Achievement, Full Professor, Department of Internal Medicine, Sackler Faculty of Medicine, Tel Aviv University, P.O.B. 39040 Ramat Aviv, Tel Aviv 69978, Israel; Director, The Gertner Institute for Epidemiology and Health Policy Research, Sheba Medical Center, Tel Hashomer, Ramat Gan 52621, Israel

Orly Tamir, MSc, MHA (orlyt@gertner.health.gov.il), Lecturer, Department of Health Management, Ariel University Center in Samaria, Ariel, Israel; Researcher, Israeli Center for Technology Assessment in Health Care, The Gertner Institute for Epidemiology and Health Policy, Research, Sheba Medical Center, Tel Hashomer 52621, Israel

Miriam Ines Siebzehner, PhD, MPA, RN (miris@ gertner.health.gov.il), Lecturer, Department of Nursing, School of Professions, Sackler Faculty of Medicine, Tel Aviv University, P.O.B. 39040, Ramat Avenue, Tel Aviv 69978; Israel; Deputy Director \& Senior Researcher, Israeli Center for Technology Assessment, in Health Care - ICTAHC, The Gertner Institute, Sheba Medical Center, Tel Hashomer, Ramat Gan 52621, Israel; Deputy Director, Division of Medical Technology Policy, Medical Technology and Infrastructure Administration, Ministry of Health, 2 Ben Tabai Street, Jerusalem 93591, Israel

\section{REFERENCES}

1. Banta D. The development of health technology assessment. Health Policy. 2003;63:121-132.

2. Banta D, Oortwijn W. Health technology assessment and health care in the European Union. Int J Technol Assess Health Care. 2000;16:626-635.

3. Bin Nun G, Katz H. International comparisons of health systems: OECD countries and Israel 1980-1988 (Hebrew). Jerusalem: Ministry of Health; 2001.

4. Givon U. Consensus conference on the treatment and rehabilitation of hip fractures in the elderly. IMAJ. 1999;1:131-136.

5. Goodman CS. HTA 101: Introduction to health technology assessment. Falls Church, VA: The Lewin Group; 2004.

6. Greenberg D, Siebzehner MI, Pliskin JS. The process of updating the National List of Health Services in Israel: Is it legitimate? Is it fair? Int J Technol Assess Health Care. 2009;25:255261. 
7. Hailey DM. Health technology assessment in Canada: Diversity and evolution. Med J Aus. 2007;187:286-288.

8. Hammerman A, Greenberg D. Estimating the budget impact of new technologies added to the national list of health services in Israel: Stakeholders' incentives for adopting a financial risksharing mechanism. Health Policy. 2009;89:78-83.

9. Israel Ministry of Health. Guidelines for long term national health policy in Israel. Jerusalem: Ministry of Health; 1989.

10. Israel Ministry of Health. Director General circular. The updating of the NLHS year 2009, No. 21/.80 Jerusalem: Ministry of Health; 2008.

11. Israel Ministry of Health. Medical Administration circular. New differential pricing, no. 25/2004. Jerusalem: Ministry of Health; 2004.

12. Jonsson E, Banta D. Management of health technologies: An international view. BMJ. 1999;319:1-3.

13. Luxenburg O, Vaknin S, Pollack G, Siebzehner M, Shemer J. Utilization patterns of CT and MRI in Israel. Harefuah. 2003;142:810-814.

14. Peleg K, Savitsky B. The effect of differential pricing for early treatment of hip fractures in elderly patients on the care provided. Oral presentation and abstracts of the 9th World Conference on Injury Prevention and Safety Promotion, Merida, Mexico, 2008.

15. Rabinovich M, Wood F, Shemer J. Impact of new medical technologies on health expenditures in Israel 2000-2007. Int J Technol Assess Health Care. 2007;23:443-448.

16. Rosen B. Health care systems in transition: Israel. In: Thomson $\mathrm{S}$, Mossialos E, eds. Health care systems in transition. Copenhagen: European Observatory on Health Care Systems; 2003.

17. Shani S, Siebzehner MI, Luxenburg O, Shemer J. Setting priorities for the adoption of health technologies on a national levelThe Israeli experience. Health Policy. 2000;54:169-185.

18. Shani S, Yahalom Z. The Israeli model for managing the National List of Health Services in an era of limited resources. Law Policy. 2002;24:133-147.

19. Shemer J, Schersten T, eds. Technology assessment in health care: From theory to practice. Jerusalem: Gefen Publishing House; 1995.

20. Shemer J, Siebzehner MI, Notzer N. A medical technology assessment course. Acad Med. 1997;72:432.

21. Shemer J, Siebzehner MI. Health technology policy in Israel at a time of health care reform. In: Shemer J, Schersten T, eds. Technology assessment in health care: From theory to practice. Jerusalem: Gefen Publishing House; 1995:42-50.

22. Shemer J, Morginstin T, Hammerman A, Luxenburg O, Shani S. Promoting medical technologies in the national list of health services: 1995-2000. Harefuah. 2003;142:82-86.

23. Shemer J. Consensus conference as a tool for national health services policy: The case for osteoporosis. IMAJ. 2000;2:375376.

24. Siebzehner MI. Shemer J. Israel's new national health insurance law. In: Shemer J, Vienonen M, eds. Reforming health care systems. Jerusalem: Gefen Publishing House; 1995:217-223.

25. Siebzehner MI. Priority setting in medical technologyevaluation of the Israeli model (Hebrew). $\mathrm{PhD}$ thesis. BenGurion University of the Negev, Department of Health Systems Management; 2004.

26. Siebzehner MI, Reuveni H, Pliskin J. Evaluation of the priority setting process for the adoption of health technologies in Israel - Opinion survey. The third International Jerusalem Conference on Health Policy, Jerusalem, Israel, 2006.

27. Siebzehner MI. Consensus statement on prevention and treatment of osteoporosis. IMAJ. 2000;2:397-401.

28. State of Israel. State Commission of Inquiry into the operation and efficiency of the healthcare system in Israel. The Majority Opinion. Jerusalem: Government Printing House; 1990.

29. Tamir O, Rabinovich M, Shani M. Year 2006 update of the Israel National List of Health Services. Isr Med Assoc J. 2006;8:595600.

30. Tal O, Tamir O, Vaknin S, et al. Technology forecasting in healthcare-Gazing into a crystal ball or a scientific tool? Poster and Abstracts of the 5th Annual Meeting of the National Institute for Health Policy Research, Tel-Aviv, Israel, 2005. 